\title{
THE PERIODIC b-EQUATION AND EULER EQUATIONS ON THE CIRCLE
}

\author{
JOACHIM ESCHER AND JÖRG SEILER
}

\begin{abstract}
In this note we show that the periodic $b$-equation can only be realized as an Euler equation on the Lie group Diff ${ }^{\infty}\left(\mathbb{S}^{1}\right)$ of all smooth and orientiation preserving diffeomorphisms on the cirlce if $b=2$, i.e. for the Camassa-Holm equation. In this case the inertia operator generating the metric on $\operatorname{Diff}^{\infty}\left(\mathbb{S}^{1}\right)$ is given by $A=1-\partial_{x}^{2}$. In contrast, the Degasperis-Procesi equation, for which $b=3$, is not an Euler equation on $\operatorname{Diff}{ }^{\infty}\left(\mathbb{S}^{1}\right)$ for any inertia operator. Our result generalizes a recent result of B. Kolev [24].
\end{abstract}

\section{INTRODUCTION}

In this note we are interested in the geometric interpretation of the socalled $b$-equation

$$
m_{t}=-\left(m_{x} u+b m u_{x}\right), \quad t \in \mathbb{R}, x \in \mathbb{S}^{1},
$$

with the momentum variable $m$ given by

$$
m=u-u_{x x},
$$

and where $b$ stands for a real parameter, cf. $[13,12,19]$. It was shown in $[13,19,26,20]$ that equation (1) is asymptotically integrable, a necessary condition for complete integrability, but only for the values $b=2$ and $b=3$. In case $b=2$ we recover the Camassa-Holm equation $(\mathrm{CH})$

$$
u_{t}-u_{t x x}+3 u u_{x}-2 u_{x} u_{x x}-u u_{x x x}=0
$$

while for $b=3$ we obtain the Degasperis-Procesi equation (DP)

$$
u_{t}-u_{t x x}+4 u u_{x}-3 u_{x} u_{x x}-u u_{x x x}=0 .
$$

Independent of (asymptotical) integrability, equation (1) possesses some hydrodynamic relevance, as described for instance in [22, 21, 11]. Each of these equations models the unidirectional irrotational free surface flow of a shallow layer of an inviscid fluid moving under the influence of gravity over a flat bed. In these models, $u(t, x)$ represents the wave's height at the moment $t$ and at position $x$ above the flat bottom.

The periodic Camassa-Holm equation is known to correspond to the geodesic flow with respect to the metric induced by the inertia operator $1-\partial_{x}^{2}$ on the diffeomorphism group of the circle, cf. [25]. Local existence of the geodesics and properties of the Riemannian exponential map were studied in $[9,10]$. The whole family of $b$-equations and in particular (DP) can be

2000 Mathematics Subject Classification. 35Q53, 58D05.

Key words and phrases. Euler equation, diffeomorphisms group of the circle, Degasperis-Procesi equation. 
realized as (in general) non-metric Euler equations, i.e. as geodesic flows with respect to a linear connection which is not necessarily Riemannian in the sense that there may not exist a Riemannian metric which is preserved by this connection, cf. [15].

Besides various common properites of the individual members of the $b$ equation, there are also significant differences to report on. It is known that solutions of the $\mathrm{CH}$-equation preserve the $H^{1}$-norm in time and that $(\mathrm{CH})$ possesses global in time weak solutions, cf. $[7,5,3]$. In particular there are no shock waves for $(\mathrm{CH})$, although finite time blow of classical solutions occurs, but in form of wave breaking: solutions to $(\mathrm{CH})$ stay continuous and bounded but their slopes may blow up in finite time, cf. [6, 8]. Wave breaking is also observed for the (DP) but in a weaker form. It seems that the $H^{1}$-norm of solutions of (DP) cannot be uniformly bounded, but $L_{\infty^{-}}$ bounds for large classes of initital values are available [16, 17, 18]. Moreover shock waves, i.e. discontinuous global travelling wave soltions are known to exist. Indeed it was shown in [14] that

$$
u_{c}(t, x)=\frac{\sinh (x-[x]-1 / 2)}{t \cosh (1 / 2)+c \sinh (1 / 2)}, \quad x \in \mathbb{R} / \mathbb{Z},
$$

is for any $c>0$ a global weak solution to the (DP) equation.

In this note we disclose a further difference between the $(\mathrm{CH})$ and the (DP) equation, by proving that in a fairly large class of Riemannian metrics on Diff ${ }^{\infty}\left(\mathbb{S}^{1}\right)$ it is impossible to realize (DP) as a geodesic flow.

The note is organized as follows. In Section 2, we first introduce the concept geodesic flows and Euler equations on a general Lie group. Subsequently, in Section 3, the important special case of $\operatorname{Diff}\left({ }^{1}\right)$ is discussed and Section 4 contains the proof of our main result.

\section{The Euler equation on a general Lie group}

In his famous article [1] Arnold established a deep geometrical connection between the Euler equations for a perfect fluid in two and three dimensions and the geodesic flow for right-invariant metrics on the Lie group of volumepreserving diffeomorphisms. After Arnold's fundamental work a lot of effort was devoted to understand the geometric structure of other physical systems with a Lie group as configuration space.

The general Euler equation was derived initially for the Levi-Civita connection of a one-sided invariant Riemannian metric on a Lie group $G$ (see [1] or [2]) but the theory is even valid in the more general setting of a one-sided invariant linear connection, see [15].

A right invariant metric on a Lie group $G$ is determined by its value at the unit element $e$ of the group, i.e. by an inner product on its Lie algebra $\mathfrak{g}$. This inner product can be expressed in terms of a symmetric linear operator $A: \mathfrak{g} \rightarrow \mathfrak{g}^{*}$, i.e.

$$
\langle A u, v\rangle=\langle A v, u\rangle, \quad \text { for all } u, v \in \mathfrak{g},
$$

where $\mathfrak{g}^{*}$ is the dual space of $\mathfrak{g}$ and $\langle\cdot, \cdot\rangle$ denotes the duality pairing on $\mathfrak{g}^{*} \times \mathfrak{g}$. Each symmetric isomorphism $A: \mathfrak{g} \rightarrow \mathfrak{g}^{*}$ is called an inertia operator on $G$. The corresponding metric on $G$ induced by $A$ is denoted by $\rho_{A}$. 
Let $\nabla$ denote the Levi-Civita connection on $G$ induced by $\rho_{A}$. Then

$$
\nabla_{\xi_{u}} \xi_{v}=\frac{1}{2}\left[\xi_{u}, \xi_{v}\right]+B\left(\xi_{u}, \xi_{v}\right)
$$

where $\xi_{u}$ is the right invariant vector field on $G$, generated by $u \in \mathfrak{g}$. Moreover, $[\cdot, \cdot]$ is the Lie bracket on $\operatorname{Vect}(G)$, the smooth sections of the tangent bundle over $G$, and the bilinear operator $B$ is called Christoffel operator. It is defined by the following formula

$$
B(u, v)=\frac{1}{2}\left[\left(\operatorname{ad}_{u}\right)^{*}(v)+\left(\operatorname{ad}_{v}\right)^{*}(u)\right],
$$

where $\left(\operatorname{ad}_{u}\right)^{*}$ is the adjoint with respect to $\rho_{A}$ of the natural action of $\mathfrak{g}$ on itself, given by

$$
\operatorname{ad}_{u}: \mathfrak{g} \rightarrow \mathfrak{g}, \quad v \mapsto[u, v] .
$$

A proof of the above statments as well as of the following proposition can be found in [15].

Proposition 1. A smooth curve $g(t)$ on a Lie group $G$ is a geodesic for a right invariant linear connection $\nabla$ defined by (3) iff its Eulerian velocity $u=g^{\prime} \circ g^{-1}$ satisfies the first order equation

$$
u_{t}=-B(u, u) \text {. }
$$

Equation (6) is known as the Euler equation.

\section{The Euler equation on Diff ${ }^{\infty}\left(\mathbb{S}^{1}\right)$}

Since the tangent bundle $T \mathbb{S}^{1} \simeq \mathbb{S}^{1} \times \mathbb{R}$ is trivial, Vect ${ }^{\infty}\left(\mathbb{S}^{1}\right)$, the space of smooth vector fields on $\mathbb{S}^{1}$, can be identified with $\mathrm{C}^{\infty}\left(\mathbb{S}^{1}\right)$, the space of real smooth functions on $\mathbb{S}^{1}$. Furthermore, the group Diff ${ }^{\infty}\left(\mathbb{S}^{1}\right)$ is naturally equipped with a Fréchet manifold structure modeled over $\mathrm{C}^{\infty}\left(\mathbb{S}^{1}\right)$, cf. [15]. The Lie bracket on $\operatorname{Vect}^{\infty}\left(\mathbb{S}^{1}\right) \simeq \mathrm{C}^{\infty}\left(\mathbb{S}^{1}\right)$ is given by ${ }^{1}$

$$
[u, v]=u_{x} v-u v_{x} .
$$

The topological dual space of Vect ${ }^{\infty}\left(\mathbb{S}^{1}\right) \simeq \mathrm{C}^{\infty}\left(\mathbb{S}^{1}\right)$ is given by the distributions $\operatorname{Vect}^{\prime}\left(\mathbb{S}^{1}\right)$ on $\mathbb{S}^{1}$. In order to get a convenient representation of the Christoffel operator $B$ we restrict ourselves to $\operatorname{Vect}^{*}\left(\mathbb{S}^{1}\right)$, the set of all regular distributions which may be represented by smooth densities, i.e. $T \in \operatorname{Vect}^{*}\left(\mathbb{S}^{1}\right)$ iff there is a $\varrho \in \mathrm{C}^{\infty}\left(\mathbb{S}^{1}\right)$ such that

$$
\langle T, \varphi\rangle=\int_{\mathbb{S}^{1}} \varrho \varphi d x \quad \text { for all } \varphi \in \mathrm{C}^{\infty}\left(\mathbb{S}^{1}\right) .
$$

By Riesz' representation theorem we may identify Vect* $\left(\mathbb{S}^{1}\right) \simeq \mathrm{C}^{\infty}\left(\mathbb{S}^{1}\right)$. In the following we denote by $\mathcal{L}_{i s}^{\text {sym }}\left(\mathrm{C}^{\infty}\left(\mathbb{S}^{1}\right)\right)$ the set of all continuous isomorphisms on $\mathrm{C}^{\infty}\left(\mathbb{S}^{1}\right)$, which are symmetric with respect to the $L_{2}\left(\mathbb{S}^{1}\right)$ inner product.

Definition 2. Each $A \in \mathcal{L}_{i s}^{\text {sym }}\left(\mathrm{C}^{\infty}\left(\mathbb{S}^{1}\right)\right)$ is called a regular inertia operator on $\operatorname{Diff}^{\infty}\left(\mathbb{S}^{1}\right)$.

\footnotetext{
${ }^{1}$ Notice that this bracket differs from the usual bracket of vector fields by a sign.
} 
Proposition 3. Given $A \in \mathcal{L}_{i s}^{\text {sym }}\left(\mathrm{C}^{\infty}\left(\mathbb{S}^{1}\right)\right)$, we have that

$$
B(u, v)=\frac{1}{2} A^{-1}\left[2 A u \cdot v_{x}+2 A v \cdot u_{x}+u \cdot(A v)_{x}+v \cdot(A u)_{x}\right]
$$

for all $u, v \in \mathrm{C}^{\infty}\left(\mathbb{S}^{1}\right)$.

Proof. Let $u, v, w \in \mathrm{C}^{\infty}\left(\mathbb{S}^{1}\right)$ be given. Recalling (5), integration by parts yields

$$
\begin{aligned}
\rho_{A}\left(\left(\operatorname{ad}_{u}\right)^{*} v, w\right) & =\rho_{A}\left(v, \operatorname{ad}_{u} w\right)=\int_{\mathbb{S}^{1}} A v \cdot\left(u_{x} w-u w_{x}\right) d x \\
& =\int_{\mathbb{S}^{1}}\left[(A v) u_{x}+((A v) \cdot u)_{x}\right] w d x
\end{aligned}
$$

This shows that

$$
\left(\operatorname{ad}_{u}\right)^{*} v=2(A v) u_{x}+u(A v)_{x} .
$$

Symmetrization of this formula completes the proof, cf. (4).

Examples 4. It may be instructive to discuss two paradigmatic examples.

(1) First we choose $A=$ id. Then $B(u, u)=-3 u u_{x}$ and the corresponding Euler equation $u_{t}+3 u u_{x}=0$ is known as the periodic inviscid Burgers equation, see e.g. $[4,23]$.

(2) Next we choose $A=$ id $-\partial_{x}^{2}$. Then the Euler equation reads as $u_{t}=-\left(1-\partial_{x}^{2}\right)^{-1}\left(3 u u_{x}-2 u_{x} u_{x x}-u u_{x x x}\right)$, which equivalent to the periodic Camassa-Holm equation, cf. (2).

\section{The FAmily of $b$-EQUATIONS}

Each $A \in \mathcal{L}_{i s}^{s y m}\left(\mathrm{C}^{\infty}\left(\mathbb{S}^{1}\right)\right)$ induces an Euler equation on Diff ${ }^{\infty}\left(\mathbb{S}^{1}\right)$. Conversely, given $b \in \mathbb{R}$, we may ask whether there exists a regular inertia operator such that the $b$-equation is the corresponding Euler equation on Diff ${ }^{\infty}\left(\mathbb{S}^{1}\right)$. We know from Example 4.2 that the answer is positive if $b=2$. The following result shows that the answer is negative if $b \neq 2$.

Theorem 5. Let $b \in \mathbb{R}$ be given and suppose that there is a regular inertia operator $A \in \mathcal{L}_{i s}^{\text {sym }}\left(\mathrm{C}^{\infty}\left(\mathbb{S}^{1}\right)\right)$ such that the b-equation

$$
m_{t}=-\left(m_{x} u+b m u_{x}\right), \quad m=u-u_{x x}
$$

is the Euler equation on $\operatorname{Diff}^{\infty}\left(\mathbb{S}^{1}\right)$ with respect to $\rho_{A}$. Then $b=2$ and $A=\mathrm{id}-\partial_{x}^{2}$.

Corollary 6. The Degasperis-Procesi equation

$$
m_{t}=-\left(m_{x} u+3 m u_{x}\right), \quad m=u-u_{x x}
$$

cannot be realized as an Euler equation for any regular inertia operator $A \in$ $\mathcal{L}_{i s}^{s y m}\left(\mathrm{C}^{\infty}\left(\mathbb{S}^{1}\right)\right)$.

Proof of Theorem 5. Let $b \in \mathbb{R}$ be given and assume that the $b$-equation is the Euler equation on $\operatorname{Diff}{ }^{\infty}\left(\mathbb{S}^{1}\right)$ with respect to $\rho_{A}$. Letting $L=1-\partial_{x}^{2}$, we then get

$$
A^{-1}\left(2(A u) u_{x}+u(A u)_{x}\right)=L^{-1}\left(b(L u) u_{x}+u(L u)_{x}\right)
$$

for all $u \in \mathrm{C}^{\infty}\left(\mathbb{S}^{1}\right)$. 
(a) Let 1 denote the constant function with value 1 . Choosing $u=1$ in (7), we get $A^{-1}\left(\mathbf{1}(A \mathbf{1})_{x}\right)=0$ and hence $(A \mathbf{1})_{x}=0$, i.e. $A \mathbf{1}$ is constant. Scaling (7), we may assume that $A \mathbf{1}=\mathbf{1}$. Next we replace $u$ by $u+\lambda$ in (7). Then we find for the left-hand side that

$$
\begin{aligned}
\frac{1}{\lambda} A^{-1} & \left(2(A(u+\lambda))(u+\lambda)_{x}+(u+\lambda)(A(u+\lambda))_{x}\right) \\
= & \frac{1}{\lambda} A^{-1}\left(2((A u)+\lambda) u_{x}+(u+\lambda)(A u)_{x}\right) \\
= & A^{-1}\left(\frac{2(A u) u_{x}+u(A u)_{x}}{\lambda}+2 u_{x}+(A u)_{x}\right) \\
& \stackrel{\lambda \rightarrow \infty}{\longrightarrow} A^{-1}\left(2 u_{x}+(A u)_{x}\right),
\end{aligned}
$$

and similarly for the right-hand side:

$$
\begin{aligned}
\frac{1}{\lambda} L^{-1} & \left(b(L(u+\lambda))(u+\lambda)_{x}+(u+\lambda)(L(u+\lambda))_{x}\right) \\
& \stackrel{\lambda \rightarrow \infty}{\longrightarrow} L^{-1}\left(b u_{x}+(L u)_{x}\right) .
\end{aligned}
$$

Combing these limits, we conclude that

$$
A^{-1}\left(2 u_{x}+(A u)_{x}\right)=L^{-1}\left(b u_{x}+(L u)_{x}\right)
$$

for all $u \in \mathrm{C}^{\infty}\left(\mathbb{S}^{1}\right)$. Setting $u_{n}=e^{i n x}$, we find that

$$
L^{-1}\left(b\left(u_{n}\right)_{x}+\left(L u_{n}\right)_{x}\right)=i \alpha_{n} u_{n}
$$

where $\alpha_{n}:=n+\left(b n /\left(1+n^{2}\right)\right)$. Applying $A$ to (8) with $u=u_{n}$ thus yields

$$
2 i n u_{n}+\left(A u_{n}\right)_{x}=i \alpha_{n} A u_{n} .
$$

Therefore $v_{n}:=A u_{n}$ solves the ordinary differential equation

$$
v^{\prime}-i \alpha_{n} v=-2 i n u_{n} .
$$

For $n \neq 0$, let us solve (9) explicitly. Assume first that $b=0$. Then

$$
v(x)=(c-2 i n x) u_{n}
$$

for some constant $c$. But this function is never $2 \pi$-periodic. Thus we must have $b \neq 0$. However, in this case

$$
v_{n}=A u_{n}=\gamma_{n} e^{i \alpha_{n} x}+\beta_{n} u_{n} \quad(n \neq 0)
$$

with $\beta_{n}=\frac{2\left(1+n^{2}\right)}{b}$ and suitable constants $\gamma_{n}$.

(b) Assume that all $\gamma_{n}$ vanish, i.e. $A u_{n}=\beta_{n} u_{n}$ for all $n \neq 0$ and $A \mathbf{1}=\mathbf{1}$. In particular, $A$ is a Fourier multiplication operator and thus commutes with $L$. Therefore we can write $(7)$ as

$$
L\left(2(A u) u_{x}+u(A u)_{x}\right)=A\left(b(L u) u_{x}+u(L u)_{x}\right) .
$$

Inserting $u=u_{n}$ a direct computation yields

$$
3\left(1+4 n^{2}\right) \beta_{n}=(1+b)\left(1+n^{2}\right) \beta_{2 n} .
$$

Using that $\beta_{n}=2\left(1+n^{2}\right) / b$, this is equivalent to $b=2$. Then $\beta_{n}=1+n^{2}$ and therefore $A$ coincides with $L$. 
(c) Let us assume there is a $p \in \mathbb{Z} \backslash\{0\}$ such that $\gamma_{p} \neq 0$. We shall derive a contradiction. Since $v_{p}=A u_{p}$ must be $2 \pi$-periodic, $\alpha_{p}$ is an integer. This implies that $b=k\left(1+p^{2}\right) / p$ for some non-zero integer $k$. We set

$$
m:=\alpha_{p}
$$

Observe that $m \neq p$, since $b \neq 0$. Thus $\left(u_{p} \mid u_{m}\right)_{L_{2}}=0$ and (10) implies that

$$
\left(A u_{p} \mid u_{m}\right)_{L_{2}}=\left(\gamma_{p} e^{i m x} \mid u_{m}\right)_{L_{2}}=\gamma_{p} .
$$

By the symmetry of $A$ we also find that

$$
\gamma_{p}=\left(A u_{p} \mid u_{m}\right)_{L_{2}}=\left(u_{p} \mid A u_{m}\right)_{L_{2}}=\overline{\gamma_{m}}\left(u_{p} \mid e^{i \alpha_{m} x}\right)_{L_{2}} .
$$

Since $\gamma_{p} \neq 0$ we must have $\gamma_{m} \neq 0$. Again by periodicity we conclude that $\alpha_{m} \in \mathbb{Z}$. But then $\alpha_{m}=p$, since otherwise we would have $\left(u_{p} \mid e^{i \alpha_{m} x}\right)_{L_{2}}=0$ and thus again $\gamma_{p}=0$. We know already that $b=k\left(1+p^{2}\right) / p$. Thus $m=\alpha_{p}=p+k$ by (11) and the definition $\alpha_{p}$. Now we calculate

$$
\begin{aligned}
p & =\alpha_{m}=\alpha_{p+k}=p+k+b \frac{p+k}{1+(p+k)^{2}} \\
& =p+k+\frac{k\left(1+p^{2}\right)}{p} \cdot \frac{p+k}{1+(p+k)^{2}},
\end{aligned}
$$

and we find

$$
p\left(1+(p+k)^{2}\right) k+k\left(1+p^{2}\right)(p+k)=0 .
$$

Observing that $k \neq 0$, an elementary calculation yields

$$
2 p^{3}+3 p^{2} k+p k+2 p+k=0 .
$$

From this we conclude that there is an $l \in \mathbb{Z}$ such that $k=p l$. With this we infer from (12) that

$$
(l+2)\left((l+1) p^{2}+1\right)=0 .
$$

The only integer solution of this equation is $l=-2$. In fact, the solution $l=-\frac{1}{p^{2}}-1$ is only possible if $p^{2}=1$ and thus again $l=-2$, since for $p^{2} \neq 1$ we have $l \notin \mathbb{Z}$. Therefore $b=-2\left(1+p^{2}\right)$ and thus $\alpha_{p}=-p$.

Moreover, we can conclude that $\gamma_{n}=0$ whenever $n \neq 0$ does not coincide with $p$ or $-p$, since otherwise the same calculation as before would show $b=-2\left(1+n^{2}\right)$ contradicting $b=-2\left(1+p^{2}\right)$.

Now insert $u=u_{p}$ in (7). The left-hand side then equals

$$
A^{-1}\left(i p \gamma_{p} \mathbf{1}-3 i p u_{2 p}\right)=i p \gamma_{p} \mathbf{1}-\frac{3 i p}{\beta_{2 p}} u_{2 p} ;
$$

for the latter identity note that $2 p$ does not coincide with $p$ or $-p$, so that $\gamma_{2 p}=0$, and hence $A^{-1} u_{2 p}=u_{2 p} / \beta_{2 p}$. Note also that $\beta_{p}=-1$. For the right-hand side we get

$$
i(1+b) \frac{p\left(1+p^{2}\right)}{1+4 p^{2}} u_{2 p}
$$

Comparing both expressions we conclude that $p \gamma_{p}=0$ which is a contradiction to $p \neq 0$ and $\gamma_{p} \neq 0$. 


\section{REFERENCES}

1. V. I. Arnold, Sur la géométrie différentielle des groupes de Lie de dimension infinie et ses applications à l'hydrodynamique des fluides parfaits, Ann. Inst. Fourier (Grenoble) 16 (1966), no. fasc. 1, 319-361. MR 0202082

2. V. I. Arnold and B. Khesin, Topological methods in hydrodynamics, Applied Mathematical Sciences, vol. 125, Springer-Verlag, New York, 1998. MR 1612569

3. A. Bressan and A. Constantin, Global conservative solutions of the Camassa-Holm equation, Arch. Rational Mech. Anal. 183 (2007), 215-239. MR 2278406

4. J. M. Burgers, A mathematical model illustrating the theory of turbulence, Advances in Applied Mechanics, Academic Press Inc., New York, N. Y., 1948, edited by Richard von Mises and Theodore von Kármán,, pp. 171-199. MR 0027195

5. A. Constantin and J. Escher, Global weak solutions for a shallow water equation, Indiana Univ. Math. J. 47 (1998), 1527-1546. MR 1687106

6. - Wave breaking for nonlinear nonlocal shallow water equations, Acta Math. 181 (1998), no. 2, 229-243. MR 1668586

7. _ Well-posedness, global existence, and blowup phenomena for a periodic quasilinear hyperbolic equation, Comm. Pure Appl. Math. 51 (1998), no. 5, 475-504. MR 1604278

8. - On the blow-up rate and the blow-up set of breaking waves for a shallow water equation, Math. Z. 233 (2000), no. 1, 75-91. MR 1738352

9. A. Constantin and B. Kolev, On the geometric approach to the motion of inertial mechanical systems, J. Phys. A 35 (2002), no. 32, R51-R79. MR 1930889

10. _ Geodesic flow on the diffeomorphism group of the circle, Comment. Math. Helv. 78 (2003), no. 4, 787-804. MR 2016696

11. A. Constantin and D. Lannes, The hydrodynamical relevance of the Camassa-Holm and Degasperis-Procesi equations, Arch. Ration. Mech. Anal. 192 (2009), no. 1, 165186. MR 2481064

12. A. Degasperis, D. D. Holm, and A. N. I. Hone, A new integrable equation with peakon solutions, Teoret. Mat. Fiz. 133 (2002), no. 2, 170-183. MR 2001531

13. A. Degasperis and M. Procesi, Asymptotic integrability, Symmetry and perturbation theory (Rome, 1998), World Sci. Publ., River Edge, NJ, 1999, pp. 23-37. MR 1844104

14. J. Escher, Wave breaking and shock waves for a periodic shallow water equation, Philos. Trans. R. Soc. Lond. Ser. A Math. Phys. Eng. Sci. 365 (2007), no. 1858, 2281-2289. MR 2329148

15. J. Escher and Kolev, The Degasperis-Procesi equation as a non-metric Euler equation, preprint, arXiv:0908.0508, 2010.

16. J. Escher, Y. Liu, and Z. Yin, Global weak solutions and blow-up structure for the Degasperis-Procesi equation, J. Funct. Anal. 241 (2006), no. 2, 457-485. MR 2271927

17. Shock waves and blow-up phenomena for the periodic Degasperis-Procesi equation, Indiana Univ. Math. J. 56 (2007), no. 1, 87-117. MR 2305931

18. J. Escher and Z. Yin, Well-posedness, blow-up phenomena, and global solutions for the b-equation, J. Reine Angew. Math. 624 (2008), 51-80. MR 2456624

19. A. N. W. Hone and J. P. Wang, Prolongation algebras and Hamiltonian operators for peakon equations, Inverse Problems 19 (2003), no. 1, 129-145. MR 1964254

20. R. I. Ivanov, On the integrability of a class of nonlinear dispersive waves equations, J. Nonlinear Math. Phys. 12 (2005), no. 4, 462-468. MR 2171998

21. W Water waves and integrability, Philos. Trans. R. Soc. Lond. Ser. A Math. Phys. Eng. Sci. 365 (2007), no. 1858, 2267-2280. MR 2329147

22. R. S. Johnson, The classical problem of water waves: a reservoir of integrable and nearly-integrable equations, J. Nonlinear Math. Phys. 10 (2003), no. suppl. 1, 72-92. MR 2063546

23. T. Kato, The Cauchy problem for quasi-linear symmetric hyperbolic systems, Arch. Ration. Mech.Anal. 58 (1975), 181-205.

24. B. Kolev, Some geometric investigations on the Degasperis-Procesi shallow water equation, Wave Motion 46 (2009), 412-419. 
25. S. Kouranbaeva, The Camassa-Holm equation as a geodesic flow on the diffeomorphism group, J. Math. Phys. 40 (1999), no. 2, 857-868. MR 1674267

26. A. V. Mikhailov and V. S. Novikov, Perturbative symmetry approach, J. Phys. A 35 (2002), no. 22, 4775-4790. MR 1908645

Institute for Applied Mathematics, University of Hannover, D-30167 HanNOVER, GERMANY

E-mail address: escher@ifam.uni-hannover.de

Department of Mathematical Sciences, Loughborough University, LeicesTERSHIRE LE11 3TU, UNITED KINGDOM

E-mail address: J.Seiler@lboro.ac.uk 